\title{
20 años de investigación en salud laboral, ...sostiene Archivos (1998-2018)
}

\author{
F.G. Benavides ${ }^{a}$, A.M. Garcia ${ }^{b}$, E. Ronda
}

DOI: 10.12961/aprl.2018.21.02.2

En este número nos gustaría celebrar con todos vosotros, lectores y lectoras de Archivos de Prevención de Riesgos Laborales, las dos primeras décadas de vida de nuestra revista. Para ello, nada mejor que empezar por el contexto, que nunca se debe olvidar, pues son muchas los cambios que se han producido durante estos 20 años. El primero, que nos afecta directamente, es la consolidación de la prevención de riesgos laborales. Si bien es cierto que con muchas limitaciones -sobre todo en la última década, consecuencia de la crisis financiera y económica-, la existencia de estructuras preventivas en una gran mayoría de las empresas, acompañadas por unas administraciones que han renovado sus estructuras para su seguimiento y control, es una realidad tangible. Basta echar una ojeada al informe del Departament d'Ocupació de la Generalitat de Catalunya de $2017^{1}$ o al de la Inspección de Trabajo del Ministerio de Trabajo $^{2}$, para palpar esta realidad. Con limitaciones, por supuesto, pero ahí está. Nos queda pendiente hacer una evaluación más detallada, para lo que solicitamos la ayuda de los y las investigadoras.

Un poco más lejano, pero cada día más próximo -las luces largas son necesarias para conducirnos-, observamos que el mundo ha cambiado, el planeta es otro, visto con la perspectiva de estos 20 años. Leyendo el informe preliminar de este pasado septiembre sobre el Futuro del Trabajo ${ }^{3}$ de la OIT, nos sobrecoge la velocidad con la que está mudando el mundo del trabajo, consecuencia de lo que ellos llaman los drivers, como son la globalización económica, los avances tecnológicos, la fragmentación de las cadenas globales de producción y el envejecimiento de la población. Ello unido a la mutación de las relaciones laborales en estos años de crisis en Europa ${ }^{4}$, con una pérdida de la capacidad de la negociación colectiva y el declinar del papel de los sindicatos. Estamos no ante una época de cambios sino ante un cambio de época, y no sabemos cómo será el trabajo en los próximos diez años, aunque como dice el Director de la OIT, ese futuro no está escrito y depende de lo que seamos capaces de hacer nosotros hoy ${ }^{3}$. De eso se trata, de gobernar nuestro presente, y Archivos es una plataforma magnífica que nos ha ayudado y nos ayuda en esta tarea.

Lo hecho desde Archivos durante estos 20 años ya ha sido reseñado en la editorial del número anterior ${ }^{5}$, y son muchos los lo-

a. Director Archivos de Prevención de Riesgos Laborales 1998-2005.

b. Directora Archivos de Prevención de Riesgos Laborales 2005-2015.

c. Directora Archivos de Prevención de Riesgos Laborales. gros. Entre ellos, y sin desmerecer lo demás, está el mismo hecho de existir. Que no es poco. Los agradecimientos son también muchos y reconocibles, y entre ellos, y de nuevo sin desmerecer a los demás, queremos aquí destacar a las autoras y autores de los 178 manuscritos originales publicados, más 63 editoriales, durante estas dos décadas. La mayoría de los originales dedicados a políticas y estrategias de prevención (19\%), vigilancia de la salud (16\%), enfermedades de origen laboral (15\%) y exposiciones laborales (10\%). Nos fijamos en ellos porque son los protagonistas de la misión genuina de una revista científica como Archivos: difundir el conocimiento científico útil para la práctica de la salud laboral ${ }^{6-8}$.

Y para dar protagonismo a los verdaderos actores de esta celebración, hemos previsto la publicación de comentarios a una selección de artículos publicados durante estas dos décadas en Archivos que nos sirvan de referencia para reflexionar sobre el avance en el conocimiento y de la práctica profesional en este tiempo. Los primeros, que se presentan en este número, tratan sobre una de las temáticas más frecuentes en nuestra revista: la vigilancia de salud de los trabajadores; dos artículos publicados en 2000, uno mostrando el análisis de las recaídas por enfermedades profesionales en un registro poblacional de declaración de casos ${ }^{9}$ y otro presentando un decálogo para la vigilancia de la salud ${ }^{10}$.

Para acabar esta nota editorial, de nuevo agradecer a las personas que ya han publicado -y a las que publicarán- sus artículos, editoriales, cartas, comentarios, etc., pues su esfuerzo por observar lo que hacemos, y plantear preguntas de cómo mejorarlo, es lo que permite a que Archivos siga siendo un mensajero de buenas noticias, aunque sean criticas. Por favor, ¡larga vida al mensajero!

Correspondencia:

Elena Ronda Pérez elena.ronda@ua.es 


\section{BIBLIOGRAFÍA}

1. Molinero E., Pitarque S., Montserrat J. Institut de Seguretat i Salud Laboral. Departament de Treball. Sistema Català d'Informació en Seguretat i Salut Laboral. Elaboració d'Indicadors. [Accedido 18 febrero 2018]. Disponible en: http://treball.gencat.cat/web/.content/09__seguretat_i_salut_laboral/publicacions/imatges/SISTEMA-CATALA-INFORMACIO-SEGURETAT-SALUTLABORAL_-ELABORACIO-INDICADORS.pdf.

2. Dirección General de Inspección de Trabajo y Seguridad Social. Ministerio de Trabajo y Seguridad Social. Informe anual de la Inspección de Trabajo y Seguridad Social 2016. [Accedido 18 febrero 2018]. Disponible en: http://www. empleo.gob.es/itss/ITSS/ITSS_Descargas/Que_hacemos/Memorias/Memoria_2016.pdf.

3. International Labour Organization. Inception report for the Global Commission on the future of work. [Accedido 9 enero 2018]. Disponible en: http:// www.ilo.org/global/topics/future-of-work/publications/WCMS_591502/lang-es/index.htm.

4. Caprile M., Sanz P., Rioboo Leston I., Welz C., Rodriguez Contreras R. Mapping varieties of industrial relations: Eurofound's analytical framework applied - Executive summary. [Accedido 18 febrero 2018]. Disponible en: https://www. eurofound.europa.eu/publications/executive-summary/2018/mapping-varieties-of-industrial-relations-eurofounds-analytical-framework-applied-executivesummary.

5. Ronda E., García A.M., Schlaghecke J. Archivos, mucho ya hecho y mucho por hacer. Arch Prev Riesgos Labor 2015; 18: 183-184.

6. Foncuberta L., Delclós J., Martínez J.M., León M., Varela P., Zapater R., et al. Archivos 2017: 20 años con Archivos. Arch Prev Riesgos Labor. 2018;21:7-10.

7. Benavides F.G. Una cultura científica para la prevención de riesgos laborales. Arch Prev Riesgos Labor. 1998: 5-6.

8. García A.M, Por qué revistas científicas, por qué Archivos. Arch Prev Riesgos Labor 2005; 9: 1-2.

9. Layana E., Artieda L., Beloqui A.M. Recaídas por enfermedades profesionales en un registro poblacional de declaración de casos (1989-1998). Arch Prev Riesgos Labor 2000; 3: 55-60.

10. Boix P., Benavides F.G., Soriano G., Moreno N., Roel J.M., García-Gómez M. Criterios básicos para la vigilancia de la salud de los trabajadores: decálogo sobre la vigilancia de la salud en el trabajo. Arch Prev Riesgos Labor. 2000; 3: 175-7.

\section{PERSPECTIVAS NÓRDICAS SOBRE \\ LA CIENCIA DEL TRABAJO \\ 10-12 de abril de 2018, Gotemburgo (Suecia) \\ Información: \\ Linda Vänskä, NIVA, Arinatie 3A, \\ FI-00370 Helsinki, Finlandia. \\ Tel.: +358405463277 \\ E-mail: linda.vanska@niva.org \\ Web: https://niva.org/}

\section{INNOVACIÓN EN PRL. \\ DRONES Y SU APLICACIÓN PRÁCTICA \\ EN MATERIA DE SEGURIDAD \\ Y SALUD LABORAL}

13 de abril de 2018, Lugo

Información:

Centro ISSGA de Lugo, Ronda de Fingoi, 170,

27071 Lugo, España

Tel.: 982294 300. Fax: 982294336

E-mail: issga.lugo@xunta.gal

Web: http://issga.xunta.gal/portal/contido/o-issga/

index.html

\section{LA PRL EN EL SECTOR COMERCIO. UNA VISIÓN DESDE LAS PYMES}

16 de abril de 2018, Madrid

Información:

Instituto Regional de Seguridad y Salud en el Trabajo (IRSST), C/ Ventura Rodríguez, 7. 2a ,3a ,5a y 6 planta, 28008, Madrid, España

Tel.: 9142058 05/900 713123

E-mail: catalogo@madrid.org

Web: www.madrid.org

\section{RIESGOS LABORALES}

Y SALUD REPRODUCTIVA

16-19 de abril de 2018, Malmö (Suecia)

Información:

Katja Pekkarinen, NIVA, Arinatie 3A,

FI-00370 Helsinki, Finlandia

Tel.: +358438241698

E-mail: katja.pekkarinen@niva.org

Web: https://niva.org/ 